\title{
Simulating target lesion for endoscopic submucosal dissection training in a live pig model ${ }^{1}$
}
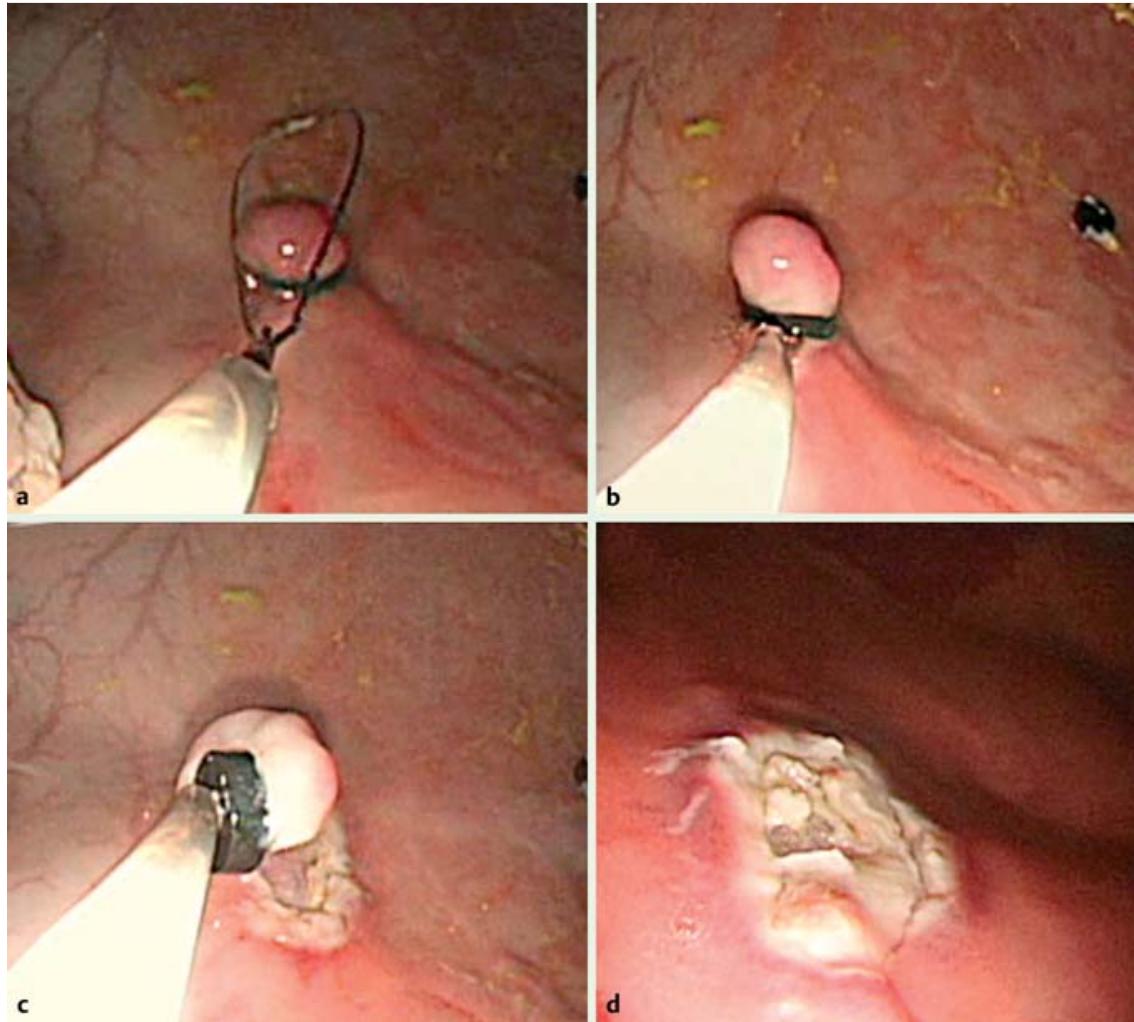

Fig. 1 A pseudopolyp was artificially created with a rubber band ligation device in a live pig stomach (a) and the snare advanced to encircle the pseudopolyp (b). The pseudopolyp was transected by snare cautery (c), leaving a mucosal defect to simulate a depressed target (d).

Endoscopic submucosal dissection (ESD) is a operator-dependent technique for performing en bloc resection of early digestive neoplasms [1]. A panel of experts from Europe reached a consensus that hands-on experience in a model of harvested pig stomach and in live pigs should be a major part of structured training [2]. In the previous training protocol, only a flat target lesion could be considered in the normal mucosa [3]. Then we developed a method of simulating target lesions in ex vivo systems [4], but this still

\footnotetext{
${ }^{1}$ This article was presented as a poster abstract at Asia-Pacific Digestive Week 2011.
}

cannot simulate a setting that provides the opportunity to respond to the potential complication of bleeding.

This study aimed to evaluate the feasibility of using a simulated target lesion in the stomach of live pig for full ESD training. A pseudopolyp was created in the upper corpus of a pig stomach using a pneumatically activated esophageal variceal ligation device (MD-48709; Sumitoma Corp., Tokyo, Japan) ( $\bullet$ Fig. 1a, b). We were able to transect the pseudopolyp with snare cautery (ESG-100, cut 1, power level 100; Olympus Optical Co., Ltd., Tokyo, Japan) ( $\bullet$ Fig. 1 c), leaving a mucosal defect to simulate a depressed target $(\diamond$ Fig. 1 d). The ESD maneuvers were similar to the standard protocol in human beings. We first used a dual knife (KD650Q; Olympus) to mark the periphery $(\bullet$ Fig.2a). The target was lifted by submucosal injection ( $\bullet$ Fig. $\mathbf{2} \mathbf{b}$ ). The mucosa outside the markings was initially pre-cut with a dual knife ( $\boldsymbol{O}$ Fig. $2 \mathrm{c}$ ) and then was incised circumferentially ( $\boldsymbol{O}$ Fig. $\mathbf{2 d}$ ) and dissected using a second-generation insulated-tip knife (KD-610L; Olympus). A Coagrasper (FD-410LR; Olympus) was used when visible vessels or active bleeding were identified ( $\bullet$ Fig. 2 e). The target lesion was then resected ( $\bullet$ Fig. $\mathbf{2 f}$ ).

The most important advantage of the model is that it could enable full ESD training, from how to make proper markings to the delineation of safety margins and the proper management of bleeding.

\section{Endoscopy_UCTN_Code_TTT_1AO_2AG}

\section{Competing interests: None}

\section{M.-J. Chen ${ }^{1,2,3}$, C.-Y. Liu ${ }^{1,2,3}$ C.-J. Chen 1,2,3, S.-C. Shih", ${ }^{1,3}$, H.-Y. Wang ${ }^{1,3}$}

${ }^{1}$ Division of Gastroenterology, Department of Internal Medicine, Mackay Memorial Hospital and Mackay Medicine, Nursing and Management College, Taipei, Taiwan

${ }^{2}$ Clinical Skills Training Center, Department of Medical Education, Mackay Memorial Hospital, Taipei, Taiwan

${ }^{3}$ Mackay Medical College, New Taipei, Taiwan 

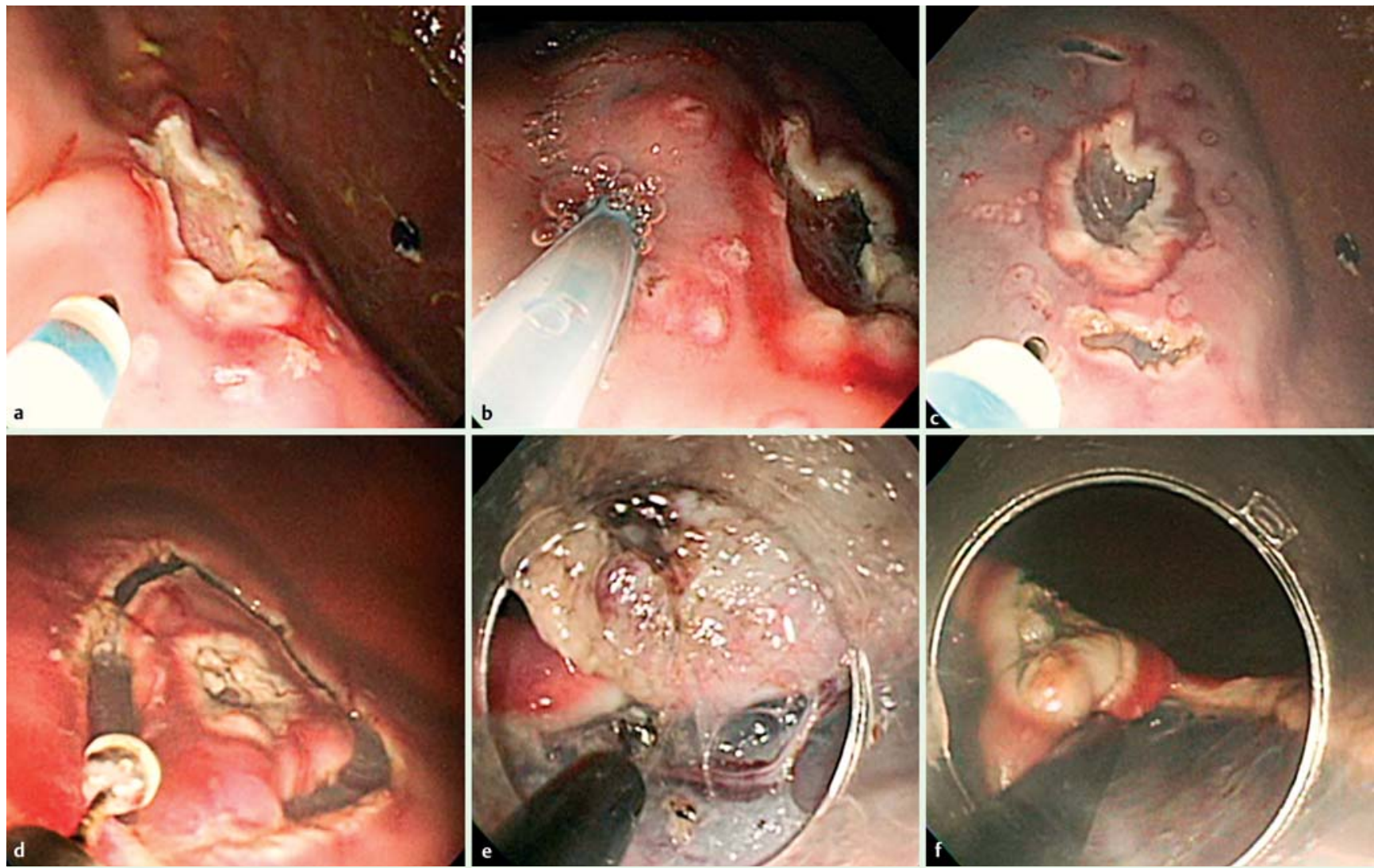

Fig.2 A dual knife was used to mark the periphery, which was set to about $5 \mathrm{~mm}$ outside of the target (a). The target was lifted by submucosal injection (b). The mucosa outside the markings was initially pre-cut with a dual knife (c). The mucosa was incised circumferentially (d) using an insulated-tip knife. A Coagrasper was used when visible vessels or active bleeding were identified (e). The target lesion was then resected (f).

\section{References}

1 Gotoda T, Yamamoto H, Soetikno RM. Endoscopic submucosal dissection of early gastric cancer. J Gastroenterol 2006; 41: 929-942

2 Deprez PH, Bergman JJ, Meisner S et al. Current practice with endoscopic submucosal dissection in Europe: position statement from a panel of experts. Endoscopy 2010; 42: $853-858$

3 Kim EY, Jeon SW, Kim GH. Chicken soup for teaching and learning ESD. World J Gastroenterol 2011; 17: 2618-2622
4 Wang TE, Wang HY, Lin CC et al. Simulating a target lesion for endoscopic submucosal dissection training in an ex vivo pig model. Gastrointest Endosc 2011; 74: 398-402

\section{Bibliography}

Dol http://dx.doi.org/

10.1055/s-0032-1309986

Endoscopy 2012; 44: E300-E301

(c) Georg Thieme Verlag KG

Stuttgart · New York

ISSN 0013-726X

\section{Corresponding author}

\section{H.-Y. Wang, MD}

Division of Gastroenterology

Department of Internal Medicine

Mackay Memorial Hospital

No. 92, Sec. 2, Chungshan North Road

Taipei

Taiwan

Fax: $+886-2-25433642$

hywang@ms2.mmh.org.tw 